\title{
Social Interaction is Unnecessary for Hindgut Microbiome Transmission in Honey Bees: the Effect of Diet and Social Exposure on Tissue-Specific Microbiome Assembly
}

Kirk E. Anderson ( $\square$ kirk.anderson@usda.gov ) USDA-ARS PWA: USDA-ARS Pacific West Area https://orcid.org/0000-0002-9846-2603

Vincent A. Ricigliano

USDA-ARS PWA: USDA-ARS Pacific West Area

Duan Copeland

University of Arizona, Tucson

Brendon M. Mott

USDA-ARS PWA: USDA-ARS Pacific West Area

Patrick Maes

University of Arizona, Tucson

\section{Research Article}

Keywords: Mycobiota, Microbiota, Sociality, Honey bee, Gut bacteria, Fungi, Hive environment, Beebread, Pollen

Posted Date: December 1st, 2021

DOI: https://doi.org/10.21203/rs.3.rs-1093978/v1

License: (c) (i) This work is licensed under a Creative Commons Attribution 4.0 International License.

Read Full License 
LRH: Kirk E. Anderson et al.

RRH: Honey bee microbiome assembly

Title: Social interaction is unnecessary for hindgut microbiome transmission in honey bees: The effect of diet and social exposure on tissue-specific microbiome assembly

Authors: Kirk E. Anderson ${ }^{1, *}$, Vincent A. Ricigliano ${ }^{1,2}$, Duan C. Copeland ${ }^{3}$, Brendon M. Mott ${ }^{1}$ and Patrick Maes ${ }^{4}$

1 ARS-USDA Carl Hayden Bee Research Center, Tucson, AZ 85719

2 Present Address: ARS-USDA Honey Bee Breeding Genetics and Physiology Research, Baton Rouge, LA 70820

3 Department of Microbiology, School of Animal \& Comparative Biomedical Sciences; University of Arizona, Tucson, AZ, USA 85721

4 Department of Entomology and Center for Insect Science University of Arizona, Tucson, AZ 85721

*Corresponding author. Email: Kirk.Anderson@usda.gov

Orchid ID: 0000-0002-9846-2603 85719. Phone: (520) 820-7186 Fax: (520) 670-6493.

\section{Co-author emails}

Vincent Ricigliano: Vincent.Ricigliano@usda.gov

30 Patrick Maes: pmaes@email.arizona.edu

31 Duan Copeland Duan.Copeland@usda.gov

32 Brendon M. Mott: Brendon.Mott@usda.gov

Key Words: Mycobiota, Microbiota, Sociality, Honey bee, Gut bacteria, Fungi, Hive

36 environment, Beebread, Pollen

\section{Acknowledgments}

38 The authors acknowledge members of the Anderson lab for technical assistance. Work funded 39 by Research Plan Anderson 2022-21000-021-00D. The USDA is an equal opportunity employer 40 and provider. 
Abstract

Honey bees are a model for host-microbial interactions with experimental designs evolving towards conventionalized worker bees. Research on gut microbiome transmission and assembly has examined only a fraction of factors associated with the colony and hive environment. Here we studied the effects of diet and social isolation on tissue-specific bacterial and fungal colonization of the midgut and two key hindgut regions. We found that both treatment factors significantly influenced early hindgut colonization explaining similar proportions of microbiome variation. In agreement with previous work, social interaction with older workers was unnecessary for core hindgut bacterial transmission. Exposure to natural eclosion and fresh stored pollen resulted in gut bacterial communities that were taxonomically and structurally equivalent to those produced in the natural colony setting. Stressed diets of no pollen or autoclaved pollen in social isolation resulted in decreased fungal abundance and bacterial diversity, and atypical microbiome structure and tissue-specific variation of functionally important core bacteria. Without exposure to the active hive environment, the abundance and strain diversity of keystone ileum species Gilliamella apicola was markedly reduced. These changes were associated with significantly larger ileum microbiotas suggesting that extended exposure to the active hive environment plays an antibiotic role in hindgut microbiome establishment. We conclude that core hindgut microbiome transmission is facultative horizontal with 5 of 6 core hindgut species readily acquired from the built hive structure and natural diet. Our findings contribute novel insights into factors influencing assembly and maintenance of honey bee gut microbiota and facilitate future experimental designs.

\section{Introduction}

A variety of symbiotic microbial associations have developed in the guts of insects and other animals. Social insects in particular present unique opportunities to investigate hostmicrobiome interactions due to variation in individual behavior, phenotype, diet, and lifespan occurring within the same genetic unit [1-3]. Given the long co-evolutionary relationship between honey bees and their microbiota, the small number of interacting taxa, and the predictability of hind gut microbiota structure, this highly social insect group has become a model for microbiome research [4-6]. However, the social and "built structure" context of honey bee life history [7] is problematic for many experiments making it difficult to gauge individual vs. group effects. Removing the honey bee individual from the colony and hive environment is necessary to test many hypotheses, but such manipulations alter a variety of biotic and abiotic influences on individual physiology, behavior and hindgut microbiota colonization $[8,9]$. In this contribution, we examine assembly of the honey bee hindgut microbiome manipulating diet and social context. We discuss the evolutionary implications of our findings and provide perspective for the interpretation and refinement of honey bee microbiome experiments.

81

Honey bee (Apis mellifera) colonies are adaptively organized groups of individuals that collect and process floral-derived nutrition via age-based division of labor [10]. Younger adults consume a diet of stored pollen and convert this nutrient source to a fat and protein rich jelly

84 used to feed developing larvae and the queen $[11,12]$. Thus, young bees are called nurses. 
Foraging bees are older, collect pollen, nectar and tree resin, and have significantly lower levels of internal nutrient stores [13]. Spanning this variation in worker task and physiology, an elaborate network of high-frequency trophallactic interactions (mouth to mouth transfer of jelly and sugar rich liquids) intimately connects the nutritional state of the colony and also facilitates microbial transmission [14]. Hive substances produced or collected by honey bees exhibit varying degrees of antimicrobial and antioxidant activities including salivary and hypopharygeal gland secretions, royal jelly, nectar, honey, and stored pollen [12, 15-19]. The core hindgut bacteria are detected consistently throughout all of these niches [20-23]. Although coprophagy has been suggested as a transmission mechanism for core gut bacteria, honey bees do not perform this behavior $[8,9]$. However, newly emerged bees immediately sample the hive environment with their mouthparts, an activity that includes cleaning the cells of recently emerged adults, and autogrooming [9, 24-26]. Pollen consumption occurs from 2-10 days of age concurrent with nursing behavior and exposure to much of the social and hive environment $[26,27]$.

Considering the honey bee gut microbiome from the colony perspective, the interactions among and between individuals and the hive environment are comprised of numerous biotic and abiotic factors influencing one another to varying degrees. This arrangement of social interactions and built structure is considered a "factory in a fortress" [28]. Although this early model largely ignored microbes and disease, the evolution of honey bee sociality was undoubtedly subject to constant microbial challenge at multiple levels including host control of the hive environment $[15,29,30]$. The honey bee harbors a highly predictable microbiota in the hindgut, and active hive environment [17, 22, 31, 32]. For the purposes of this paper, we define the active hive microbiota as microbes typically found on the mouthparts of workers and queens and throughout the interactive network of food distribution, including the social stomach (crop), the hypopharygeal glands, stored honey, stored pollen (beebread), and larval environments. The core hindgut bacteria are prevalent throughout the active hive environment but differ in prevalence by niche [22, 23, 33, 34]. This foundation aids in distinguishing disease transmission from hindgut microbiome transmission and microbes vectored from the pollination environment.

The hindgut of the adult worker honey bee harbors a core hindgut microbial community of five omnipresent bacterial groups totaling approximately $10^{8}-10^{9}$ bacterial cells [31, 35]. The hindgut microbiome is demarcated by the pylorus, where metabolic waste materials are excreted by the Malpighian tubules into the hindgut environment. Bacterial membership and function differs by hindgut region. The pylorus of healthy bees is often colonized by a sixth bacterium, Frischella perrara, causing an immune cascade and melanized scab [36, 37]. Snodgrassella alvi interfaces with host epithelium at the ileum, and occurs in a biofilm with Gilliamella apicola, and Lactobacillus firm 5 [4, 38, 39]. The rectum comprises the largest bacterial community and is dominated primarily by four species of Lactobacillus firm 5 , and lesser amounts of Lactobacillus firm 4 and Bifidobacterium. Less understood, and sporadically detected in the worker hindgut are Lactobacillus kunkeei and Bombella apis (a synonym of Parasaccharibacter apium), bacteria found with greater abundance/prevalence in queen guts and/or the active hive environment $[17,23,40,41]$.

In worker bees, the establishment of a typical hindgut microbiota happens in the first few days of adult life, leads to increased weight gain, reduced susceptibility to pathogens, and 
129 priming of the host immune system [4, 42, 43]. However, atypical or altered bacterial

130 establishment in early adult life leads to reduced weight, impaired development, disease progression and early mortality [21,44]. Studies investigating adult hindgut colonization have produced variable results, and the contribution of various factors to hindgut microbiota establishment remains unclear $[8,9,35,45,46]$. Larvae shed their gut lining prior to pupation, and are recolonized as adults. Although studies are limited, few microbes are thought to survive this transition [47]. One primary difficulty for experimental design is that adult hindgut assembly happens concurrently with the consumption of stored pollen by newly emerged worker bees. Following adult emergence, honey bees experience multiple social cues, pollen consumption, and exposure to a variety natural hive niches that might affect microbiome establishment $[17,26]$.

Most recently, the honey bee hindgut microbiota has become the subject of significant research interest due to its role in disease susceptibility [32, 43], a major role played by gut bacteria in most host species. Here we explore hindgut microbiome assembly (transmission and establishment) of the adult worker bee considering social and non-social environments, fungal colonization and diet. We allowed natural eclosion from the natal frame, and assessed microbiome colonization of nine-day-old worker bees for specific gut regions (midgut, ileum and rectum). We used bacterial $16 \mathrm{~S}$ rRNA gene sequencing, fungal specific $18 \mathrm{~S}$ sequencing and qPCR to quantify treatment effects on microbiota colonization and character.

\section{Methods}

We gathered emerging brood frames from 16 actively growing colonies in July 2016, at the Carl Hayden Bee Research Center in Tucson AZ. Capped brood frames containing a high proportion of dark-eyed pupae were placed into screened collection boxes under controlled climate conditions [48]. We allowed natural ecolsion, defined as newly emerged workers (NEWs) chewing out of their natal cocoon and capped cell, and performing early life behaviors on their natal wax frame including larval cell cleaning $[9,26]$. All NEWs were exposed to naturally occurring hive components beebread, honey, wax frame and empty development cells for 0-3 hours.

A single cohort of newly emerged worker (NEWs) containing approximately 3000 bees were marked with a paint dot on their thorax, and divided among healthy full-sized colonies or nuc-box cages containing different diet treatments and two 19X9.125 inch frames. To control for the active hive environment, we returned 100 marked NEWs to each of three healthy growing colonies. We placed 300 marked NEWs into each of 9 nuc-box cages (three treatments with 3 replicates), and provided the following diet treatments: freshly collected and stored pollen (beebread), autoclaved corbicular pollen, and no pollen (sucrose syrup only). Thus, exposure to the active hive environment only occurred for the colony control. The beebread diet treatment consisted of one frame with abundant freshly-collected pollen obtained near the center of the brood nest from healthy colonies that were actively collecting and storing pollen. In choice tests, workers prefer 1-2 day-old pollen [49], so we provided fresh pollen that was likely collected that day or the previous day, as indicated by the lack of a honey cap, as is typical of older beebread. Both the colony control and fresh beebread diets are referred to as natural diets. 
The autoclaved pollen treatment was comprised of corbicular pollen pellets stripped

174 from the hind legs of foragers using a pollen-trapping device attached to the front of active

175 hives. To the corbicular pollen we added $10 \%$ sterile $\mathrm{H}_{2} \mathrm{O}(\mathrm{w} / \mathrm{v})$ then worked the mixture into a

176 pollen paste. This mixture was autoclaved and packed into a frame of empty wax comb. The no

177 pollen treatment received a frame of empty wax comb. All non-social cages were given a 2nd

178 19X9.125" frame of empty drawn wax comb to encourage clustering and provided sterilized

$17970 \%$ sucrose syrup and sterile water ad libitum via $30 \mathrm{ml}$ drip bottles and maintained at $35^{\circ} \mathrm{C}$

180 and $50 \%$ relative humidity. Both the autoclaved pollen and no pollen (sucrose only) diets are

181 referred to as stressed diets. After nine days, bees from each cage or colony were dissected and

182 processed as described in [48].

183

184

\section{PCR and MiSeq}

For $\mathrm{N}=48$ ileum and $\mathrm{N}=48$ rectum samples, we amplified the $\mathrm{V} 6-\mathrm{V} 8$ variable region of the 16S rRNA gene using PCR primers 799F (acCMGGATTAGATACCCKG + barcode) and bac1193R (CRTCCMCACCTTCCTC). DNA was amplified using the HotStarTaq Plus Master Mix Kit (Qiagen, USA) with the following thermocycler program: $94{ }^{\circ} \mathrm{C}$ for $3 \mathrm{~min}$, followed by 28 cycles of $94{ }^{\circ} \mathrm{C}$ for $30 \mathrm{~s}, 53{ }^{\circ} \mathrm{C}$ for $40 \mathrm{~s}$ and $72{ }^{\circ} \mathrm{C}$ for $1 \mathrm{~min}$, with a final elongation step at $72{ }^{\circ} \mathrm{C}$ for 5 min. PCR products were confirmed on a $2 \%$ agarose gel. PCR products were then used to prepare DNA libraries via the protocol for Illumina MiSeq DNA library preparation. Sequencing was performed at the University of Arizona Genetics Core on a MiSeq following the manufacturer's guidelines.

\section{$\underline{\text { MiSeq sequence analysis }}$}

Sequences were processed using MOTHUR v.1.35.1 [50]. The make.contigs command was used to join forward and reverse reads. We then removed the last five base pairs of the amplicon using the SED command in UNIX. We removed sequences containing ambiguous bases using the screen.seqs command, and unique sequences were generated using the unique.seqs command. A count file containing group information was generated using the count.seqs command. Sequences were aligned to Silva SSUREF database (v102 [51]) using the align.seqs command. Sequences not overlapping in the same region and columns not containing data were removed using the filter.seqs command. Sequences were pre-clustered using the pre.cluster command. Chimeras were removed using UCHIME [52] and any sequences that were not of known bacterial origin were removed using the remove.seqs command. All remaining sequences were classified using the classify.seqs command. All sequences with only one or two (single/doubletons) associated reads were removed using the AWK command in UNIX. A distance matrix was constructed for the aligned sequences using the dist.seqs command. Sequences were classified with the RDP Naive Bayesian Classifier [53] using a manually constructed training set containing sequences sourced from the greengenes 16S rRNA database (version gg_13_5_99 accessed May 2013 [54]), the RDP version 9 training set, and all full length honeybee-associated gut microbiota listed in NCBI (accessed July 2013). Operational taxonomic units (OTUs) were generated using the cluster command. Representative sequences for each OTU were generated using the get.oturep command. To further confirm taxonomy, resulting representative sequences were subject to a BLAST query using the NCBI nucleotide database. 
Bacterial and Fungal Quantification

Total bacteria and fungi in three gut niches were quantified using the BactQuant and FungiQuant qPCR primers $[55,56]$ in a SYBR-green assay on Bio-Rad CFX96 thermocyclers. To provide absolute quantification of $16 \mathrm{~S}$ or $18 \mathrm{~S}$ rDNA copy number and ensure inter-run comparability, in-run standard curves and no-template controls were included on each run. Plasmid standards for each assay were created using either a $16 \mathrm{~S}$ or $18 \mathrm{~S}$ gene clone (using Invitrogen $\mathrm{pCR}^{\circledR} 2.1-\mathrm{TOPO}^{\mathrm{TM}}$ cloning vector (\#K4500-40) and $\mathrm{DH} 5 \alpha^{\mathrm{TM}}$ cells (\#18265017) per manufacturer's specifications), purified via plasmid mini-prep kit (Thermo Scientific \#K0503), dsDNA/ $\mu$ l was determined via Implen NanoPhotometer P300, and the known mass of plasmid plus PCR insert was used to calculate $16 \mathrm{~S}$ or $18 \mathrm{~S}$ plasmid standard copies per $\mu$ l. Standard curves were calculated from a 10-fold serial dilution of the plasmid standards included on each run.

230

Statistical analysis

We calculated microbiota diversity by treatment and niche, using unique sequences identified in the bioinformatics pipeline. Using the summary.single command in Mothur, we rarefied to the smallest library $(19,427$ for ileum's and rectum's and 54,288 for the hindgut as a whole). We calculated observed number of unique sequences, Shannon's $(H)$, the Effective Number of Species or richness (ENS- defined as the $\exp (\mathrm{H})$ ) and equitability or evenness of the microbiota defined as H/Hmax, where Hmax = In(total \# of OTU's). We quantified the top 196 unique OTUs, defining them by species, proportional representation and core hindgut membership.

The microbiome data set was transformed by bactiquant results and species-specific rRNA copy number. To incorporate community size in the analysis, we multiplied the proportional abundance of OTUs returned by amplicon sequencing by the total bacterial $16 \mathrm{~S}$ rRNA gene copies determined with qPCR for each individual tissue type. All core bacterial genomes contain four 16S rRNA gene copies except Lactobacillus kunkeei (5), Bifidobacterium asteroides (2) and Bombella apis (1). OTUs representing non-core diversity were summed and corrected for community size via mean (4.2) 16S rRNA gene copy number [57]. To allow the use of parametric multivariate analyses [58], we converted bacterial relative abundance to ratios among all OTUs [59] using the software CoDaPack's centered log-ratio (CLR) transformation [60]. The analysis of centered log ratios are a measure of ratio abundance of a single taxon relative to the rest of the microbiota, so the data analysis reflects changes in the structure of the microbiota with respect to a single taxon. Principle component analysis (PCA) bi-plots were generated to explore the variance in the data. We performed a MANOVA comparing the hindgut (ileum and rectum) microbiota by treatment, corrected for multiple comparisons. We examined the CLR transformed data using two separate MANOVA models. The first analyzed the data as 4 separate treatments with colony or box nested in treatment. The second analyzed as the data as two diet treatments; normal diet (colony and pollen consumed the same diet) and perturbed diet, a group defined by significantly decreased consumption of fats, proteins and complex carbohydrates.

As a more straightforward measure of change in particular taxa without respect to other community members, we compare estimated cell counts by niche and taxon using an ANOVA 
261 performed on log transformed cell counts normalized for species-specific 16S rRNA gene copy

262 number. Effects attributed to diet were examined by comparing stressed diets (autoclaved

263 corbicular pollen or no pollen) to normal diets of freshly collected beebread (colony and pollen). Microbial community structure was also compared with diet source and social context as factors. We compared bacterial and fungal copy number by niche using one-way ANOVA (Tukey HSD post-hoc) and two-sample t-tests. We performed correlations examining log transformed fungal abundance with each major bacterial taxon. All analyses were conducted in either JMP_v11(JMP_ 1989-2007) and/or SAS_v9.4 (SAS Institute Inc., Cary, NC).

\section{Results}

Microbial community analysis

Next generation sequencing returned 4,236,606 quality trimmed reads (400 bp) for the 96 libraries, an average of 44,131 sequences per library (Table S1). To distinguish $F$. perrara from $G$. apicola, we clustered the sequences at $99 \%$ similarity, producing a total of 4367 OTUs following the exclusion of singletons and doubletons. We then confirmed taxonomy via NCBI BLAST and consolidated core gut phylotypes from the top 40 OTUs reducing the data set to 8 core gut phylotypes. Following consolidation and taxonomic confirmation, eight of the top 40 unique OTUs $(16,22,26,30,32,33,35$, and 36) were sparse containing a high frequency of zero values. These eight OTUs were consolidated into a 9th group consisting of "other" ( $\Sigma$ OTUs 4197 plus the eight listed above). Following consolidation, the eight phylotypes and "other" represented $97 \%$ and $1.5 \%$ of the total sequences respectively, and were used for downstream statistical analyses. The dependent variable "other" is a combination of OTUs representing a measure of diversity abundance for each tissue.

Microbiota and Mycobiota size

Considering the three treatments where diet consumption could be measured, gut weight differed significantly by diet treatment, and was correlated positively with microbial community size across treatments ( $R s q=.29, p=0.0007$ ). In the treatment provided only sucrose solution, the guts of nine day old adults weighed significantly less than the treatments provided pollen in some form, and newly emerged adults consumed $36 \%$ less autoclaved pollen than they did freshly-collected pollen [48].

Bacterial abundance differed by treatment in 2 of 3 gut tissues (Fig. 1). Relative to the colony control, bacterial load was significantly greater in the midgut $\left(F_{3,44}=3.1, p=0.04\right)$, and ileum $\left(F_{3,44}=7.9, p=0.0002\right)$ of workers fed a diet of fresh beebread but raised in social isolation. In the midgut, bacterial abundance differed between the colony control and fresh pollen treatment (post-hoc test, $p=0.02$ ). In the ileum, the colony control differed in bacterial abundance from fresh pollen $(p=0.001)$, and no pollen $(p=0.0005)$. Bacterial abundance in the rectum did not differ by treatment.

Fungal abundance differed by treatment across all three gut sections. In natural colony "control" bees, mycobiome size decreased significantly from midgut to ileum to rectum, and was significantly negatively associated with microbiota size considering the gut as a whole (Fig. 2). Relative to the colony control, fungal load decreased significantly in the midgut, ileum and rectum of bees fed fresh pollen. In the ileum and rectum, this difference was more pronounced

304 for bees fed autoclaved pollen or no pollen (Fig. 2). Fungal abundance differed most drastically 
in the midgut $\left(F_{3,44}=64.3, p<0.0001\right)$ as compared to the other two gut regions; Post-hoc tests revealed that colony control midguts differ from those of fresh pollen, autoclaved pollen and no pollen $(p<0.0001)$, and the fresh pollen treatment also differed from autoclaved pollen $(p<0.005)$ and no pollen $(p<0.0004)$. Fungal abundance also differed among treatments in the ileum $\left(F_{3,44}=90.8, p<0.0001\right)$; Post-hoc tests show colony control differs from fresh pollen ( $p$ $<0.0004)$, autoclaved pollen and no pollen $(p<0.0001)$, and fresh pollen differs from autoclaved pollen and no pollen $(p<0.0001)$. In the rectum, differences were significant but less pronounced $\left(\mathrm{F}_{3,44}=8.6, p=0.0001\right)$. Following post-hoc tests, fungal abundance in rectums of the colony control differed from fresh pollen $(p<0.002)$ autoclaved pollen $(p<0.0004)$ and no pollen $(p<0.0009)$.

\section{Microbiota diversity}

Based on unique bacterial OTUs, the diversity of combined hindgut tissues (ileum and rectum) differed by treatment including observed species, Shannon's $\mathrm{H}$, and Equitability, a measure of evenness (Fig. 3). Considering the detection of all unique OTUs, the colony control showed the greatest number of species, effective species, and evenness, followed by fresh pollen, autoclaved pollen and no pollen in that order. Considering the top 196 unique OTUs, herein referred to as "establishment", the richness of Gilliamella apicola differed by social environment (Table 1). Hindguts from the fresh pollen treatment contained significantly fewer established OTUs than did the colony control: (18 vs. 47; one-tailed test of proportions Z = $5.6 \mathrm{p}$ $<0.00001)$. The richness of the other four core hindgut groups did not differ by social environment, but $S$. alvi richness differed by diet treatment showing 21-22 established strains in normal diets, but only 11-13 in stressed diets (Table 1).

\section{OTU differences by treatment}

Colony control- NEWs that we returned to the colony environment $<3$ hours after emergence developed a typical gut microbiota characterized by evenness of the core microbiota in both the ileum and rectum (Fig. 3 and 4). All of the core species were present in relative and absolute abundance typical of a nurse worker sampled from the natural hive environment. The microbiota was characterized by high equitability among core species including core ileum species F. perrara and G. apicola. Fungi were strongly associated with microbiota structure and taxonomy throughout the gut of the colony control (Fig. 2). The mycobiota and microbiota showed strong associations throughout the alimentary tract, with fungi decreasing in absolute abundance moving towards the rectum concurrent with increasing microbiota density. The occurrence and evenness of Frischella perrara was associated with "bioactive" pollen consumption in both the colony control and fresh-stored pollen treatment (Fig. 4).

Fresh-stored pollen- Relative to the colony control, newly emerged bees removed from social/hive contact but provided a diet of fresh-stored pollen showed significantly larger bacterial communities in the midgut and ileum, and changes to both absolute and relative abundance of core OTUs (Fig. 5). Found primarily in the ileum, and important for gut function, $G$ apicola was abundant in only one of three fresh pollen replicates. However, with the exception of $G$. apicola, the taxonomy and relative abundance of all other core hindgut species resembled that of the colony control (Fig. 4). In the ileum, L. firm5 increased in both relative and absolute 
abundance while G. apicola and total fungi decreased (Fig. 5, Table S2). The rectum microbiota of the fresh pollen treatment was almost indistinguishable from that of the colony control, but fungi was significantly reduced, and the fungal-bacterial association was disrupted throughout the alimentary tract relative to the colony control (Figs. 1 and 2).

Autoclaved pollen- We designed this treatment to provide pollen nutrients, but exclude any biotic activity from the pollen itself, or microbes in the corbicular pollen pellet. Thus, it is a control of sorts for the fresh pollen diet. However, the consumption of autoclaved pollen was significantly less (63\%) than that of fresh-stored pollen [48], and subsequently, microbiota size in the ileum was significantly associated with diet consumption, measured as gut weight. Even so, with the consumption of solid food, the microbiota still established in a tissue-specific manner, with the ileum composition differing from that of the rectum (Fig. 4). Relative to the colony control, this treatment resulted in similar sized microbiotas, but significantly smaller mycobiotas throughout the gut (Fig. 1). Both absolute and relative abundance of core OTUs differed from the control including significant increases in the ileum of L. firm5, L. kunkeei, and Bo. apis, and decreases of $G$ apicola, S. alvi and F. perrara (Figs. 4 and 5, Table S2). G apicola was virtually absent from this treatment establishing in only a single individual. When compared to the fresh-beebread diet, differences were similarly pronounced for both hindgut tissues (Table S2).

No pollen- With brief exposure to the colony environment but no pollen nutrition, size of the midgut, ileum and rectum microbiotas did not differ from the colony control (Fig. 1). This treatment differed taxonomically from the control and other treatments for many core OTUs, including L. firm5, L. kunkeei, G. apicola and Bo. apis, in the ileum and L. firm 4 and B. asteroides in the rectum (Figs. 4 and 5, Table S2). In general, the taxonomy and structure of the ileum microbiota was similar to that of the rectum, and the diversity indices indicate less diverse, dominance communities relative to the colony control and other treatments (Fig. 3).

\section{Discussion}

Our results build on previous findings [8, 9, 35], and suggest that both diet and active hive exposure are critical for generational transmission and microbiota assembly (Figs. 1 and 2). Workers excluded from natural eclosion and frame exposure, then fed sterile pollen in sterile environments established hindgut microbiotas too small for next generation sequencing, suggesting little vertical transmission and poor establishment [8]. Here we demonstrate that natural eclosion, brief exposure to the emergence frame, and a diet of fresh beebread resulted in the assembly of adult hindgut microbiomes highly similar to those that established in the active hive environment (Figs 4 and 5). The primary difference when excluding the active hive environment was a sharp decline in fungal abundance, and a 50\% failure of Gilliamella apicola to establish in the ileum. Establishment G. apicola may require extended exposure to the active hive so the host and incipient microbiota can "sample" multiple strains of G. apicola compatible with themselves, and the pioneer gut residents that establish prior to G. apicola; L. firm5 and S. alvi $[8,9]$.

Below we argue that core hindgut microbiome transmission in honey bees is not reliant on social transmission, but is best defined as facultative horizontal because it occurs readily in the absence of older workers, and is acquired largely via diet and emergence frame exposure 
392 (Fig. 4). In general, our hypothesized transmission mode resembles that of the marine environment [62], because it appears difficult for NEWs to avoid horizontal transmission from the hive environment. The near obligate mode in marine environments, horizontal transmission is considered beneficial for the maintenance of host-symbiont interactions because it creates competition among symbionts and allows partner choice, including mechanisms of host reward and punishment [63]. Based on mathematical models, partner choice is predicted to require horizontal transmission so hosts (and perhaps early symbionts) can choose between subsequent symbionts [64]. Because no single experiment is definitive, we discuss our results broadly with careful attention to experimental context and design applied in separate studies. Given the nature of our results, we discuss the potential associations of fungi with microbiota assembly, and two major distinctions suggested by the dependent variables; a comparison of social to asocial environments, and natural to stressed diets.

\section{Transmission and Assembly}

406 The two major experiments testing vertical transmission between honey bee worker generations concluded that social interaction of NEWs with older workers either had little effect, or did not produce a typical hindgut microbiome $[8,9]$. Our present results agree and suggest that NEWs acquire their core hindgut bacteria from natural eclosion, frame exposure and the consumption of fresh beebread $[17,20]$. Foragers likely inoculate fresh beebread with the core hindgut bacteria while packing pollen into corbiculae and while unloading pollen into the hive storage cell. Foragers have defecated at least once, but the frequency during pollen collection is unknown. The simple act of pollen collection may move continual trace amounts of pollen through the gut [65] where they are inoculated with core bacteria. Foragers gather pollen by cleaning it off their body hairs with fine-toothed leg combs often contacting the distal abdomen. This process can require hundreds of repetitions to load a single forager with gathered pollen. Honey bee workers do not practice proctodeal (mouth to cloaca) trophallaxis [26], but during flight, freshly collected pollen is first mixed with honey and hypopharyngeal gland secretions before it is packed into the corbiculae using the mouthparts and a series of leg to leg transfers. The entire suite of core gut bacteria can be found on the mouthparts, and in the hypopharyngeal gland of older bees [21, 23].

Returning newly emerged bees to the active hive environment provides contact with various hive substances related to local and hive-specific biotic conditions, microorganisms, secretions and behaviors, known disease and opportunists. Because newly emerged worker bees perform emergence behaviors as part of their life history, all treatments were allowed natural eclosion and brief ( $<3 \mathrm{hrs}$ ) exposure to the emergence frame. Given this initial exposure, the asocial fresh pollen treatment was highly similar to the social colony control based on multiple measures (Figs. 1-5). In the stressed and sterilized diet treatments, we detected significantly less fungal abundance and bacterial diversity (Figs. 1 and 3). Under normal conditions, newly emerged adult worker bees chew out of their pupal casings, clean cells from the larval nest area, then consume pollen or available nutrition $[9,26]$. Following this exposure, both diet and social isolation significantly affected the establishment of hindgut microbiota explaining similar amounts of variation (Figs 4 and 5, Table S2). In agreement with past work $[8,44]$, the greatest diversity and evenness occurred in the colony control, but total richness and evenness of the fresh-pollen treatment did not differ significantly from the control 
colonies (Fig. 3). This suggests that the majority of the hindgut microbiota is readily acquired by NEWs sampling a small fraction of the available hive space then consuming freshly-collected pollen. While transmission can occur primarily via these activities, the factors that govern microbiome assembly apparently rely on extended exposure to the active hive environment. Early life behaviors are often critical for microbiome establishment and subsequent health in animals [66], and behavior of honey bee workers separated from the active hive environment is abnormal, confounding attempts to distinguish social vs. hive exposure.

We analyzed the top 196 OTUs as a metric of establishment, and found that the fresh pollen treatment contained a greater variety of core hindgut OTUs than did bees from the control colony with the single exception of G. apicola (Table 1 ). This suggests that transmission was similar, but the process of assembly differed. When bees consumed fresh beebread in social isolation, five of six core hindgut species had similar patterns of prevalence, occurrence and diversity in the hindgut. Only the establishment of $G$. apicola seemed to rely on extended exposure to the active hive. Moreover, we observed that only ileums containing two to four unique $G$. apicola sequences seemed to establish at all regardless of treatment, suggesting that G. apicola establishes best as an interspecies partnership or that various $G$. apicola strains are partitioned by niche or resource availability. With a diet of fresh pollen in social isolation, S. alvi bloomed in the pylorus/ ileum increasing by nearly an order of magnitude relative to the colony control. S. alvi growth is suppressed by G. apicola in vitro, [67], perhaps explaining in part the increase in $S$. alvi abundance associated with limited exposure to the active hive environment (Fig. 5).

S. alvi is functionally associated with G. apicola, and the abundance of these two species correlate strongly and predictably in the ileum of normal colony-reared bees [68] (Fig. 4). S. alvi is associated with the gut lining of the ileum, where it is hypothesized to consume and mitigate host supplied oxygen [4,38]. Under normal conditions, or when colonized by a "conventional" microbiota, the central region of the ileum becomes anoxic whereas oxygen remains detectable in the center and periphery of "germ free" bee ileums fed sterile pollen [4]. Data on foragers suggests this relationship may be variable or change with age or diet [69]. Strongly allied with the host epithelium, S. alvi contributes to biofilm life, including SCFA and siderophore production $[38,70]$. Based on our analysis of the top 200 unique OTUs and other work, diversity of $G$. apicola strains is deep relative to its abundance in the hindgut system suggesting strong competition for partnership with S. alvi (Table 1). Confirming earlier hypotheses [68], the significant abundance of established G. apicola variants relative to the other core members suggests that the early ileum ecosystem and/or $S$. alvi specifically selects for $G$. apicola diversity of function (Table 1). Correspondingly, G. apicola strains show notable variation across many functional categories including oxidative stress and carbohydrate utilization, while $S$. alvi strains are typically fixed for similar functions indicating competition and host fidelity respectively [38, $48,71-73]$.

Confirmed in separate laboratory experiments, both S. alvi and G. apicola establish poorly when NEWs were exposed to either 5 or 300 older siblings and wax frames of unstated origin $[8,9]$. Even when directly feeding isolated NEWs with fresh macerated adult hindgut contents mixed with pollen, $G$. apicola established poorly relative to the colony control, attaining typical proportions in $<50 \%$ of individuals [8], mirroring our results for fresh-stored pollen (Figs. 4 and 5). Collectively these results suggest that $G$. apicola establishment is 
sensitive to a socially related factor. Positively correlated with the abundance of G. apicola, fungal density was significantly diminished throughout the gut, suggesting that fungi are also difficult to acquire with limited exposure to the active hive environment. More control of microbial exposure is required to test this hypothesis. If this particular bacterial partnership does not establish, it appears to trigger a cascade of negative changes in host physiology [21, 44]. Exposure to a greater variety of $G$. apicola strains will increase the chance of compatibility with sister strains, and ileum partner S. alvi. Below we discuss patterns in the dependent variables associated with this failed partnership.

G. apicola and/or the ileum partnership with S. alvi may be functionally "replaced" by $L$. kunkeei and Bo. apis under various environmental conditions (Fig. 4), and more generally across studies of microbiota variation examining diet, stress and social exposure [32]. L. kunkeei and Bo. apis populate the queen ileum and tend to co-occur with a positive association across many honey bee niches $[3,23,74]$. Although their abundance in worker guts is associated with host deficiencies, these species may buffer highly pathogenic microbial changes in the gut. Bo. apis and $L$. kunkeei are sugar fermenting aerobes, negatively associated with fungal density in the rectum and ileum respectively (Table S2, Fig. 6). Their abundance in the hindgut may indicate oxygen availability, a dysbiotic condition. Based on the results of a companion study examining enzyme properties of these same samples [48], the dysbiotic workers consuming stressed diets suffered significant depletions of head gland proteins, a critical marker of development in 9-day old worker bees. This represents a significant cost to the colony, because NEWs cannot perform the duties of a young nurse, and will likely contribute little resources as an older forager. Perhaps most informative, this entire nutritional microbiota dynamic was strongly related to fungal abundance, a factor long ignored in microbiome studies of the honey bee gut and other organisms (Figs 1 and 2).

504

505

\section{Fungal relationships}

The variation in our results attributable to diet (fig. 1) indicates that a fungal inoculum is obtained from exposure to the active hive environment and hive-stored pollen (Figs. 1 and 2). The significant depletion of gut fungi in the stressed diet treatments was associated with a variety of revealing microbiome changes (Fig. 3). Although not explicitly tested, we are confident that bacteria and fungi present the stressed diets were killed by autoclaving, consistent with our results for microbiota density and diversity (Figs. 1 and 3). Bo. apis was abundant and diverse in the dysbiotic ileum microbiomes resulting from sterilized and stressful diet conditions (Figs. 4 and 5). Across the data set, Bo. apis became abundant when fungi were sparse suggesting competitive release. In support of this, Bo. apis is a fungal antagonist demonstrated to inhibit the growth of Aspergillus flavus, a mycotoxin producing mold ubiquitous in beebread [75]. Similarly, Bo. apis abundance across the data set was negatively associated with all three core ileum species F. perrara, G. apicola and S. alvi (Table S2). Notably, these are the bacteria hypothesized to require social transmission by earlier investigations [8, 9]. For comparison, the ileum of the honey bee queen is a low fungus environment that supports Bo. apis, L. kunkeei and Lactobacillus firm5, and occasionally S. alvi, but not G. apicola, or F. perrara [3]. Both L. kunkeei and Bo. apis were significantly more abundant in the stressed diet treatments. Diet is the difference between worker and queen phenotypes [28], suggesting microbiome and mycobiome evolution in the gut were sculpted in part by this factor. 
524 Collectively, patterns in the dependent variables combined with known bacterial and gut

525

526

527

528

529

530

531

532

533

534

535

536

537

538

539

540

541

542

543

544

545

546

547

548

549

550

551

552

553

554

555

556

557

558

559

560

561

562

563

564

565

566

567 function suggest that fungi or fungal associated factors contribute to core-hindgut microbiota assembly especially in the ileum (Fig. 2). Our data are also consistent with resident yeasts in the worker gut [47] inhibiting the establishment of Bo. apis under natural diet conditions (Fig. $6)$, although this remains to be tested.

Fungi and bacteria are considered competitors for simple plant-derived substrates, and antagonistic and mutualistic interactions have evolved for recalcitrant substrates found in pollen such as cellulose and lignin. Aerobic cellulose degradation is ubiquitous among fungi whereas anaerobic degradation of cellulose is common among bacteria that inhabit the anoxic digestive tracts of animals [76]. A vast variety of fungi occur in hive-stored pollen and floral nectar including osmotolerant yeasts that briefly bloom in 1-2 day-old fresh pollen [77]. Although fungi were rare in our samples, they were strongly associated with keystone ileum species, G. apicola (Fig. 6), a symbiont with functional diversity for recalcitrant pollen substrates [78]. Saccharomycetes are known to colonize the nurse bee gut [46], and some appear to be vertically transmitted, surviving pupation to proliferate in adults [47]. Recent work on the first pass infant meconium in humans suggests that the mycobiota also begins at birth, and despite their rarity, fungi are involved in critical host functions and opportunistic disease [79].

The collection of recent results supports the existence of a rare fungal community that populates the gut of honey bee workers [46, 47,69]. In agreement with another recent study [69] we found a strong relationship between bacterial and fungal density throughout the alimentary tract in the colony control, perhaps a reflection of broad changes in gut $\mathrm{pH}$ and oxygen (Fig. 2). As a bioindicator of oxygen availability in the colony control, fungal density decreased and bacterial density increased approaching the rectum concurrent with the shift from aerobic to anaerobic metabolism [70]. Within each gut niche however, bacterial and fungal abundance were positively associated suggesting a lack of antagonism. This relationship was pronounced in the colony control, lost in the fresh pollen treatment, and reversed in the stressed diet conditions wherein fungi was most abundant in the rectum (Figs. 1 and 2). While these patterns suggest a shift in gut physiology [4,69], more detailed experiments are required to test this hypothesis. Results from foragers indicate variable oxygen availability in the rectum [69], but core bacterial genome function suggests resilience to these fluctuations [80].

The rectum microbiota of NEWs fed normal diets was almost indistinguishable from that of the colony control, establishing similarly regardless of ileum changes (Fig. 5). The rectum communities associated with stressed diets featured atypical representation of core species with reduced abundance of both fungi and core bacteria Lactobacillus firm4 and Bifidobacterium (Fig. 1 and 5). Across the data set, Fungi associated positively with these OTUs suggesting synergy of function in the hindgut (Table S2). Almost exclusive to the rectum of healthy worker bees, Bifidobacterium is a facultative anaerobe equipped to exploit a large swath of recalcitrant polysaccharides considered prebiotic carbohydrates that support the maintenance of healthy gut flora $[48,78,81]$. Polysacharride enzyme activity was significantly depleted in the hindguts of NEWs fed the stressed diet treatments (Ricigliano et al. 2017), and Bifidobacterium, a ubiquitous fermentative constituent of healthy gut flora, was largely absent in half of the samples (Fig. 5).

How does fresh pollen consumption affect microbiota establishment? Relative to the fresh pollen fed treatments, the lack of solid food (sugar only) resulted in a sporadic pattern of 
species co-occurrence and increased microbiota similarity between in the ileum and rectum

569 (Figs. 4-5), suggesting that the tissue specificity of hindgut colonization may depend in part on

570 the consumption of high nutrient solids (pollen or pollen substitute). Consumption of the autoclaved pollen diet treatment produced gut communities intermediate between fresh pollen and sterile sucrose syrup. Despite hydrating and autoclaving, workers ate the autoclaved pollen diet at $63 \%$ of the fresh pollen diet [48]. The pollen (or solid) diet may affect peristalic motility, or the production of midgut peritrophic matrix, processes that mitigate microbiome establishment in other model insects [82]. Freshly-stored pollen is enzymatically active, releasing carbohydrate-active enzymes involved in plant cell wall modification into the bee digestive tract upon consumption [48]. Similarly, recent work suggests that fresh pollen consumption is associated with the release of reactive oxygen species [65], conditions that may favor the establishment of the core hindgut bacteria [83]. In addition to limiting microbial exposure, autoclaving bee collected pollen certainly altered the palatable properties of pollen including $\mathrm{pH}$, solute concentration and pollen vitality. Similar to a previous study, natural diets were associated with uniform $F$. perrara establishment and niche specificity for the pylorus [36], associations completely lost in the stressed diet treatments (see also [8]). While S. alvi, G. apicola and $F$. perrara all populate the pylorus [36], fungal load had the strongest relationship with $F$. perrara abundance (Spearmans $=0.48, p<0.001$ ), again highlighting the potential contribution of fungal associated factors to microbiome assembly. environment and is readily acquired with natural eclosion $[9,17,20,84]$. It also dominates the hindgut microbiota and is a functionally variable phylotype thought to include at least four gut prevalent species (Brochet et al. 2021). Based on detailed genome analysis from two locations, four functionally different species of the Lactobacillus firm5 phylotype are abundant in every hindgut, and hypothesized to coexist via resource partitioning [85]. Although less precise, our unique sequence analysis based on distinct gut regions supports the coexistence of 2-4 major strains of firm 5 in the hindgut, but also suggests that Lactobacillus firm 5 may associate by niche. While our $400 \mathrm{bp}$ sequence cannot distinguish among three of the four major Lactobacillus firm 5 species, OTU2 corresponds to Lactobacillus apis which appears to colonize first [9], and preferentially colonize the ileum under normal diet conditions. This suggests $L$. apis niche fidelity for the ileum, perhaps via adaptations to exploit host-excreted nutrients. Lactobacillus apis -host fidelity was disrupted in the stressed diet treatments but the same group of unique Lactobacillus firm5 sequences dominated the hindgut in a different way; stressed diet treatments tended towards greater evenness of Lactobacillus firm5 species, while microbiotas associated with normal diets tended towards greater dominance.

\section{Conclusion}

606 be a more robust evolutionary strategy because it promotes increased population variability, allows for partner choice, and results in greater competition within and between species/strains [63]. Many factors, including a high degree of strain variability [86] are consistent with transmission from various active hive refugia followed by assembly that relies on partner choice. The competition for transmission and survival in the hive environment likely promotes an ever-changing pool of functional strain variants. If exposure to various competing 
612 strains leads to greater microbiome competence, then mechanisms like built structures that

613 expand and diversify transmission space would be beneficial [62]. Facilitated horizontal

614 transmission may occur in many host species that build extended structures as part of their life

615 histories [7]. In honey bees, the hive itself might be considered the maternal microbiome, a

616 transmission source filled with the complete hindgut microbiome signature. As hypothesized

617 [8], the presence of active hive related factors may represent a form of social immunity, limiting 618 microbiota size and modulating membership in the hindgut, particularly the ileum.

619 While many microbes are introduced from the pollination environment, the active hive

620 environment does not represent an alien microbiome composed of non-specialized residents; it

621 is comprised in large part of microbes carried with worker bees when they reproduce by

622 budding $[20,83]$. These colony microbes then populate the built hive environment. When filled

623 with stored food and developing young, the hive environment provides a variety of niche

624 characteristics similar to those encountered in gut environments including $\mathrm{pH}$, oxidative and

625 osmotic stress, and various antimicrobial agents [16, 17, 87]. This built environment allows

626 selection to operate simultaneously on an extended pool of population variants, yet retain a

627 level of fidelity required for host-symbiont co-evolution. As an example, the two primary

628 species of bacteria that dominate the hive environment, L. kunkeei and Bo. apis, are also core

629 species that colonize the queen ileum $[3,40,88]$. Newly emerged queen bees do not contact

630 their mother queen, so by deduction, the single most important member of the hive acquires

631 their gut microbiota before/during adult emergence and/or via the hive environment.

632

633

Our results show that hindgut microbiota assembly involves both fungi and bacteria, and relies on factors associated with diet and active hive exposure. With few exceptions, fungi

634 have been ignored in studies of the honey bee microbiota $[46,47]$. Symbiotic relationships between Hymenoptera and fungi are numerous [89], and the relationship between bacteria and fungi in the gut is an emerging area of study [90]. Based on our findings, it seems quite likely that the much smaller fungal microbiota co-occurs with the bacterial biofilm, and interacts with the host and bacterial microbiota as in other exemplars [91].

\section{References}

641

642

1. Kwong WK, Medina LA, Koch H, et al (2017) Dynamic microbiome evolution in social

644 bees. Sci Adv 3:e1600513. https://doi.org/10.1126/sciadv.1600513

645

646

647

2. Engel P, Martinson VG, Moran NA (2012) Functional diversity within the simple gut microbiota of the honey bee. Proc Natl Acad Sci U S A 109:11002-11007.

648 https://doi.org/10.1073/pnas.1202970109

3. Anderson KE, Ricigliano VA, Mott BM, et al (2018) The Queen Gut Refines with Age:

649

650 Longevity Phenotypes in a Social Insect Model. bioRxiv 1-16. https://doi.org/10.1101/297507

651

652

653

654

655

4. Zheng H, Powell JE, Steele MI, et al (2017) Honeybee gut microbiota promotes host weight gain via bacterial metabolism and hormonal signaling. Proc Natl Acad Sci U S A 114:4775-4780. https://doi.org/10.1073/pnas.1701819114

5. Zheng H, Steele MI, Leonard SP, et al (2018) Honey bees as models for gut microbiota research. Lab Anim (NY) 47:317-325. https://doi.org/10.1038/s41684-018-0173-x

6. Moran N a. (2015) Genomics of the honey bee microbiome. Curr Opin Insect Sci 
656

657

658

659

660

661

662

663

664

665

666

667

668

669

670

671

672

673

674

675

676

677

678

679

680

681

682

683

684

685

686

687

688

689

690

691

692

693

694

695

696

697

698

699

700

701

10:22-28. https://doi.org/10.1016/j.cois.2015.04.003

7. Turner JS (2005) Extended Physiology of an Insect-Built Structure. 36-38

8. Powell JE, Martinson VG, Urban-Mead K, Moran N a. (2014) Routes of Acquisition of the Gut Microbiota of the Honey Bee Apis mellifera. Appl Environ Microbiol 80:73787387. https://doi.org/10.1128/AEM.01861-14

9. Anderson KE, Rodrigues PAP, Mott BM, et al (2016) Ecological Succession in the Honey Bee Gut: Shift in Lactobacillus Strain Dominance During Early Adult Development. Microb Ecol 71:1008-1019. https://doi.org/10.1007/s00248-0150716-2

10. Huang ZY, Robinson GE (1996) Regulation of honey bee division of labor by colony age demography. Behav Ecol Sociobiol 39:147-158.

https://doi.org/10.1007/s002650050276

11. Amdam G V., Norberg K, Hagen A, Omholt SW (2003) Social exploitation of vitellogenin. Proc Natl Acad Sci 100:1799-1802. https://doi.org/10.1073/pnas.0333979100

12. Ohashi K, Natori S, Kubo T (1997) Change in the mode of gene expression of the hypopharyngeal gland cells with an age-dependent role change of the worker honeybee Apis mellifera L. Eur J Biochem 249:797-802

13. Crailsheim K (1990) The Protein Balance of the Honey Bee Worker. Apidologie 21:417-429

14. Crailsheim K, Franzens DK, Graz A- (1992) The flow of jelly within a honeybee colony. J Comp Physiol B 162:681-689. https://doi.org/10.1007/BF00301617

15. Evans JD, Spivak M (2010) Socialized medicine: individual and communal disease barriers in honey bees. J Invertebr Pathol 103:S62--S72

16. Kwakman PHS, te Velde A a, de Boer L, et al (2010) How honey kills bacteria. FASEB J 24:2576-82. https://doi.org/10.1096/fj.09-150789

17. Anderson KE, Carroll MJ, Sheehan TIM, Mott BM (2014) Hive-stored pollen of honey bees: many lines of evidence are consistent with pollen preservation , not nutrient conversion. Mol Ecol. https://doi.org/10.1111/mec.12966

18. Fontana R, Mendes MA, De Souza BM, et al (2004) Jelleines: A family of antimicrobial peptides from the Royal Jelly of honeybees (Apis mellifera). Peptides 25:919-928. https://doi.org/10.1016/j.peptides.2004.03.016

19. Ohashi K, Natori S, Kubo T (1999) Expression of amylase and glucose oxidase in the hypopharyngeal gland with an age-dependent role change of the worker honeybee (Apis mellifera L.). Eur J Biochem 265:127-33

20. Anderson KE, Sheehan TH, Mott BM, et al (2013) Microbial ecology of the hive and pollination landscape: bacterial associates from floral nectar, the alimentary tract and stored food of honey bees (Apis mellifera). PLoS One 8:e83125. https://doi.org/10.1371/journal.pone.0083125

21. Maes PW, Rodrigues PAP, Oliver R, et al (2016) Diet-related gut bacterial dysbiosis correlates with impaired development, increased mortality and Nosema disease in the honeybee (Apis mellifera). Mol Ecol 25:5439-5450. https://doi.org/10.1111/mec.13862

22. Dalenberg H, Maes P, Mott B, et al (2020) Propolis envelope promotes beneficial bacteria in the honey bee (Apis mellifera) mouthpart microbiome. Insects 11:1-12. https://doi.org/10.3390/insects11070453 
702

703

704

705

706

707

708

709

710

711

712

713

714

715

716

717

718

719

720

721

722

723

724

725

726

727

728

729

730

731

732

733

734

735

736

737

738

739

740

741

742

743

744

745

746

747
23. Floyd AS, Mott BM, Maes P, et al (2020) Microbial ecology of european foul brood disease in the honey bee (Apis mellifera): Towards a microbiome understanding of disease susceptibility. Insects 11:1-16. https://doi.org/10.3390/insects11090555

24. Jander R (1976) Grooming and pollen manipulation in bees (Apoidea): the nature and evolution of movements involving the foreleg. Physiol Entomol 1:179-194. https://doi.org/10.1111/j.1365-3032.1976.tb00960.x

25. Crailsheim K (1998) Trophallactic interactions in the adult honeybee (Apis mellifera L.). Apidologie 29:97-112. https://doi.org/10.1051/apido:19980106

26. Seeley TD (1982) Adaptive significance of the age polyeithism schedule in honeybee colonies. Behav Ecol Sociobiol 11:287-293

27. Seeley D, Kolmes A (1991) Age Polyethism for Hive Duties in Honey Bees - Illusion or Reality? When marked with paint for behavioral studies, newly emerged honeybees return invariably to for Day. Ethology 87:284-297

28. Hölldobler B, Wilson EO (2008) The Superorganism: The Beauty, Elegance, and Strangeness of Insect Societies. W. W. Norton \& Company

29. Cremer S, Armitage SAO, Schmid-Hempel P (2007) Social Immunity. Curr Biol 17:693-702. https://doi.org/10.1016/j.cub.2007.06.008

30. Schmid-hempel P (2000) Mating, parasites and other trials of life in social insects. Microbes Infect 2:515-520. https://doi.org/10.1016/s1286-4579(00)00316-6

31. Martinson VG, Danforth BN, Minckley RL, et al (2011) A simple and distinctive microbiota associated with honey bees and bumble bees. Mol Ecol 20:619-628. https://doi.org/10.1111/j.1365-294X.2010.04959.x

32. Anderson KE, Ricigliano VA (2017) Honey bee gut dysbiosis: a novel context of disease ecology. Curr Opin Insect Sci 22:125-132. https://doi.org/10.1016/j.cois.2017.05.020

33. Maes PW, Floyd AS, Mott BM, Anderson KE (2021) Overwintering honey bee colonies: Effect of worker age and climate on the hindgut microbiota. Insects 12:116. https://doi.org/10.3390/insects12030224

34. Corby-Harris V, Maes P, Anderson KE (2014) The bacterial communities associated with honey bee (apis mellifera) foragers. PLoS One 9:e95056. https://doi.org/10.1371/journal.pone.0095056

35. Martinson VG, Moy J, Moran N a (2012) Establishment of characteristic gut bacteria during development of the honeybee worker. Appl Environ Microbiol 78:2830-40. https://doi.org/10.1128/AEM.07810-11

36. Engel P, Bartlett KD, Moran NA (2015) The bacterium frischella perrara causes scab formation in the gut of its honeybee host. MBio. https://doi.org/10.1128/mBio.00193-15

37. Emery O, Schmidt K, Engel P (2017) Immune system stimulation by the gut symbiont Frischella perrara in the honey bee (Apis mellifera). Mol Ecol 26:2576-2590. https://doi.org/10.1111/mec.14058

38. Powell JE, Leonard SP, Kwong WK, et al (2016) Genome-wide screen identifies host colonization determinants in a bacterial gut symbiont. Proc Natl Acad Sci U S A 113:13887-13892. https://doi.org/10.1073/pnas.1610856113

39. Martinson VG, Moy J, Moran NA (2012) Establishment of characteristic gut bacteria during development of the honey bee worker. Appl Environ Microbiol 78:28302840. https://doi.org/10.1128/AEM.07810-11 
748

749

750

751

752

753

754

755

756

757

758

759

760

761

762

763

764

765

766

767

768

769

770

771

772

773

774

775

776

777

778

779

780

781

782

783

784

785

786

787

788

789

790

791

792

793

40. Tarpy DR, Mattila HR, Newton ILG (2015) Characterization of the honey bee microbiome throughout the queen-rearing process. Appl Environ Microbiol AEM.00307-15. https://doi.org/10.1128/AEM.00307-15

41. Vojvodic S, Rehan SM, Anderson KE (2013) Microbial Gut Diversity of Africanized and European Honey Bee Larval Instars. PLoS One 8:e72106.

https://doi.org/10.1371/journal.pone.0072106

42. Kwong WK, Mancenido AL, Moran NA (2017) Immune system stimulation by the native gut microbiota of honey bees Subject Category : Subject Areas : 1-9

43. Raymann K, Moran NA (2018) The role of the gut microbiome in health and disease of adult honey bee workers. Curr Opin Insect Sci 26:97-104. https://doi.org/10.1016/j.cois.2018.02.012

44. Schwarz RS, Moran NA, Evans JD (2016) Early gut colonizers shape parasite susceptibility and microbiota composition in honey bee workers. Proc Natl Acad Sci U S A 113:9345-9350. https://doi.org/10.1073/pnas.1606631113

45. Dong Z, Li H, Chen Y, et al (2020) Colonization of the gut microbiota of honey bee ( Apis mellifera ) workers at di ff erent developmental stages. Microbiol Res 231:126370. https://doi.org/10.1016/j.micres.2019.126370

46. Yun J, Jung M, Kim PS, Bae J (2019) Social status shapes the bacterial and fungal gut communities of the honey bee. Sci Rep 1-11. https://doi.org/10.1038/s41598-01819860-7

47. Tauber JP, McMahon D, Ryabov E V., et al (2021) Honeybee intestines retain low yeast titers, but no bacterial mutualists, at emergence. Yeast 1-13. https://doi.org/10.1002/yea.3665

48. Ricigliano VA, Fitz W, Copeland DC, et al (2017) The impact of pollen consumption on honey bee (Apis mellifera) digestive physiology and carbohydrate metabolism. Arch Insect Biochem Physiol 96:1-14. https://doi.org/10.1002/arch.21406

49. Carroll MJ, Brown N, Goodall C, et al (2017) Honey bees preferentially consume freshlystored Pollen. PLoS One 12:1-21. https://doi.org/10.1371/journal.pone.0175933

50. Schloss PD, Westcott SL, Ryabin T, et al (2009) Introducing mothur: open-source, platform-independent, community-supported software for describing and comparing microbial communities. Appl Environ Microbiol 75:7537-41. https://doi.org/10.1128/AEM.01541-09

51. Pruesse E, Quast C, Knittel K, et al (2007) SILVA: a comprehensive online resource for quality checked and aligned ribosomal RNA sequence data compatible with ARB. Nucleic Acids Res 35:7188-7196

52. Edgar RC, Haas BJ, Clemente JC, et al (2011) UCHIME improves sensitivity and speed of chimera detection. Bioinformatics 27:2194-200. https://doi.org/10.1093/bioinformatics/btr381

53. Wang Q, Garrity GM, Tiedje JM, Cole JR (2007) Naive Bayesian classifier for rapid assignment of rRNA sequences into the new bacterial taxonomy. Appl Environ Microbiol 73:5261-5267. https://doi.org/10.1128/AEM.00062-07

54. DeSantis TZ, Hugenholtz P, Larsen N (2006) Greengenes, a chimera-checked 16S rRNA gene database and workbench. Appl Environ Microbiol

55. Liu CM, Aziz M, Kachur S, et al (2012) BactQuant: An enhanced broad-coverage bacterial quantitative real-time PCR assay. BMC Microbiol 12:56. 
794

795

796

797

798

799

800

801

802

803

804

805

806

807

808

809

810

811

812

813

814

815

816

817

818

819

820

821

822

823

824

825

826

827

828

829

830

831

832

833

834

835

836

837

838

839 https://doi.org/10.1186/1471-2180-12-56

56. Liu CM, Kachur S, Dwan MG, et al (2012) FungiQuant: a broad-coverage fungal quantitative real-time PCR assay. BMC Microbiol 12:1. https://doi.org/10.1186/1471-2180-12-255

57. Větrovský T, Baldrian P (2013) The Variability of the $16 \mathrm{~S}$ rRNA Gene in Bacterial Genomes and Its Consequences for Bacterial Community Analyses. PLoS One 8:1-10. https://doi.org/10.1371/journal.pone.0057923

58. Pearson K (1986) Mathematical Contributions to the Theory of Evolution.--On a Form of Spurious Correlation Which May Arise When Indices Are Used in the Measurement of Organs. Proc R Soc London 60:489-498. https://doi.org/10.1098/rspl.1896.0076

59. Gloor GB, Reid G (2016) Compositional analysis: a valid approach to analyze microbiome high throughput sequencing data. Can J Microbiol 703:cjm-2015-0821. https://doi.org/10.1139/cjm-2015-0821

60. Comas M (2011) CoDaPack 2.0: a stand-alone, multi-platform compositional software. Options 1-10

61. SAS Institute Inc., Cary N No Title

62. Russell SL (2019) Transmission mode is associated with environment type and taxa across bacteria-eukaryote symbioses: A systematic review and meta-analysis. FEMS Microbiol Lett 366:1-13. https://doi.org/10.1093/femsle/fnz013

63. Shapiro JW, Turner PE (2014) The impact of transmission mode on the evolution of benefits provided by microbial symbionts. Ecol Evol 4:3350-3361. https://doi.org/10.1002/ece3.1166

64. Usher KM, Bergman B, Raven JA (2007) Exploring cyanobacterial mutualisms. Annu Rev Ecol Evol Syst 38:255-273.

https://doi.org/10.1146/annurev.ecolsys.38.091206.095641

65. McKinstry M, Prado-Irwin SR, Adames TR, Snow JW (2020) Retained metabolic activity in honey bee collected pollen has implications for pollen digestion and effects on honey bee health. Apidologie 51:212-225. https://doi.org/10.1007/s13592-01900703-x

66. O'Mahony SM, Marchesi JR, Scully P, et al (2009) Early Life Stress Alters Behavior, Immunity, and Microbiota in Rats: Implications for Irritable Bowel Syndrome and Psychiatric Illnesses. Biol Psychiatry 65:263-267. https://doi.org/10.1016/j.biopsych.2008.06.026

67. Ludvigsen J, Rangberg A, Avershina E, et al (2015) Shifts in the midgut/pyloric microbiota composition within a honey bee apiary throughout a season. Microbes Environ 30:235-244. https://doi.org/10.1264/jsme2.ME15019

68. Steele MI, Kwong WK, Whiteley M, Moran NA (2017) Diversification of Type VI Secretion System Toxins Reveals Ancient. MBio 8:1-19

69. Callegari M, Crotti E, Fusi M, et al (2021) Compartmentalization of bacterial and fungal microbiomes in the gut of adult honeybees. npj Biofilms Microbiomes 7:. https://doi.org/10.1038/s41522-021-00212-9

70. Kwong WK, Moran NA (2016) Gut microbial communities of social bees. Nat Rev Microbiol 14:374-384. https://doi.org/10.1038/nrmicro.2016.43

71. Zheng H, Nishida A, Kwong WK, et al (2016) Metabolism of Toxic Sugars by Strains of the Bee Gut Symbiont Gilliamella apicola. MBio 7:e01326-16. 
840

841

842

843

844

845

846

847

848

849

850

851

852

853

854

855

856

857

858

859

860

861

862

863

864

865

866

867

868

869

870

871

872

873

874

875

876

877

878

879

880

881

882

883

884

885
https://doi.org/10.1128/mBio.01326-16

72. Rothman JA, Russell KA, Leger L, et al (2020) The direct and indirect effects of environmental toxicants on the health of bumble bees and their microbiomes. bioRxiv 44:. https://doi.org/10.1101/2020.04.24.060228

73. Ludvigsen J, Porcellato D, Amdam G V., Rudi K (2018) Addressing the diversity of the honeybee gut symbiont Gilliamella: Description of Gilliamella apis sp. nov., isolated from the gut of honeybees (Apis mellifera). Int J Syst Evol Microbiol 68:1762-1770. https://doi.org/10.1099/ijsem.0.002749

74. Tarpy DR, Mattila HR, Newton ILGG (2015) Development of the honey bee gut microbiome throughout the queen-rearing process. Appl Environ Microbiol 81:3182-3191. https://doi.org/10.1128/AEM.00307-15

75. Miller DL, Smith EA, Newton ILG (2020) A bacterial symbiont protects honey bees from fungal disease. bioRxiv. https://doi.org/10.1101/2020.01.21.914325

76. Leschine SB (1995) Cellulose degradation in anaerobic environments. Annu Rev Microbiol 49:399-426. https://doi.org/10.1146/annurev.mi.49.100195.002151

77. Detry R, Simon-Delso N, Bruneau E, Daniel HM (2020) Specialisation of yeast genera in different phases of bee bread maturation. Microorganisms 8:1-14.

https://doi.org/10.3390/microorganisms8111789

78. Zheng H, Perreau J, Elijah Powell J, et al (2019) Division of labor in honey bee gut microbiota for plant polysaccharide digestion. Proc Natl Acad Sci U S A 116:2590925916. https://doi.org/10.1073/pnas.1916224116

79. Wu X, Xia Y, He F, et al (2021) Intestinal mycobiota in health and diseases : from a disrupted equilibrium to clinical opportunities. 1-18

80. Bottacini F, Milani C, Turroni F, et al (2012) Bifidobacterium asteroides PRL2011 Genome Analysis Reveals Clues for Colonization of the Insect Gut. 7:1-14. https://doi.org/10.1371/journal.pone.0044229

81. Turroni F, Bottacini F, Foroni E, et al (2010) Genome analysis of Bifidobacterium bifidum PRL2010 reveals metabolic pathways for host-derived glycan foraging. Proc Natl Acad Sci U S A 107:19514-19519

82. Ha EM, Oh CT, Bae YS, Lee WJ (2005) A direct role for dual oxidase in Drosophila gut immunity. Science (80- ) 310:847-850. https://doi.org/10.1126/science.1117311

83. Anderson KE, Maes PW (2021) Gut and Colony Microbiota of Honey Bees Apis mellifera: Social Immunity, Opportunistic Disease and Survival Overwinter. Microbiome Rev Res Sq

84. Powell JE, Martinson VG, Urban-Mead K, Moran NA (2014) Routes of acquisition of the gut microbiota of the honey bee Apis mellifera. Appl Environ Microbiol 80:73787387. https://doi.org/10.1128/AEM.01861-14

85. Brochet S, Quinn A, Mars RAT, et al (2021) Niche partitioning facilitates coexistence of closely related gut bacteria. bioRxiv 41:2021.03.12.434400

86. Ellegaard KM, Engel P (2019) Genomic diversity landscape of the honey bee gut microbiota. Nat Commun 10:. https://doi.org/10.1038/s41467-019-08303-0

87. Nicolson SW (2009) Water homeostasis in bees, with the emphasis on sociality. J Exp Biol 212:429-434. https://doi.org/10.1242/jeb.022343

88. Elijah Powell J, Eiri D, Moran NA, Rangel J (2018) Modulation of the honey bee queen microbiota: Effects of early social contact. PLoS One 13:.

https://doi.org/10.1371/journal.pone.0200527 
886 89. Kaltenpoth M (2009) Actinobacteria as mutualists: general healthcare for insects? Trends Microbiol 17:529-535. https://doi.org/10.1016/j.tim.2009.09.006

\section{Statements and Declarations}

Funding: This work was funded by the USDA-ARS, research plan 305 Anderson 2022-21000-021-

901

902

903 OOD. The ARS is an equal opportunity employer and provider.

91. Nash AK, Auchtung TA, Wong MC, et al (2017) The gut mycobiome of the Human Microbiome Project healthy cohort. Microbiome 5:153. https://doi.org/10.1186/s40168-017-0373-4

904

905

Competing Interests: The authors have no relevant financial or non-financial interests to disclose.

Author Contributions: Kirk E. Anderson and Vincent A. Ricigliano conceived of and designed research. Kirk E. Anderson, Vincent A. Ricigliano, Duan C. Copeland, Brendon M. Mott and Patrick Maes performed research. Brendon M. Mott managed the project, and Kirk E. Anderson and Patrick Maes analyzed the data. Kirk E. Anderson wrote the first draft of the manuscript and all authors commented on successive manuscript versions. All authors read and approved the final submission.

918

919

920

921

922

923

924

925

926

927

928

929

Data availability: Next gene sequencing libraries of the ileum and rectum were deposited in GenBank under Sequence Read Archive (SRA) submission number SUB10690181.

Ethics approval: This study involved non-threatened invertebrate species. The honey bees sacrificed in this study were treated humanely. 
931 Table 1. Number of unique core hindgut OTUs establisheda in the hindgut by treatment.

932

\begin{tabular}{|c|c|c|c|c|c|c|c|c|c|c|}
\hline 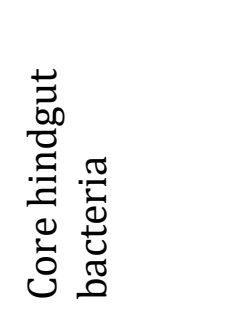 & 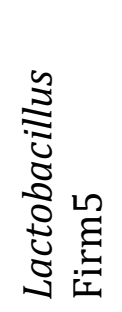 & 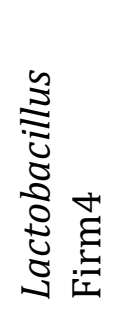 & 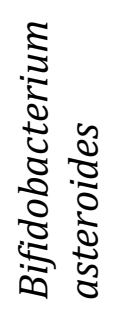 & 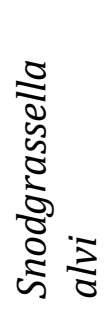 & 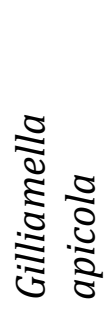 & 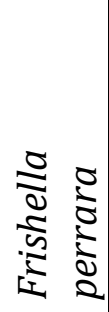 & 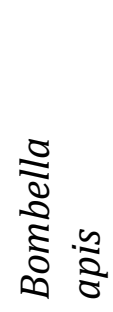 & 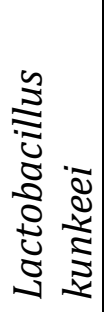 & 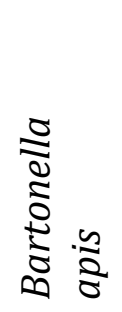 & $\begin{array}{l}\dot{\Phi} \\
\stackrel{ \pm}{0}\end{array}$ \\
\hline Total & 41 & 6 & 20 & 27 & 55 & 4 & 18 & 12 & 2 & 11 \\
\hline $\begin{array}{l}\text { Colony } \\
\text { Control }\end{array}$ & 23 & 3 & 11 & 21 & 47 & 4 & 9 & 7 & 1 & 7 \\
\hline $\begin{array}{l}\text { Fresh } \\
\text { Pollen }\end{array}$ & 28 & 4 & 16 & 22 & $18^{*}$ & 4 & 10 & 7 & 1 & 4 \\
\hline $\begin{array}{l}\text { Autoclaved } \\
\text { pollen }\end{array}$ & 29 & 5 & 12 & $11^{\#}$ & $10^{*}$ & 3 & 13 & 10 & 1 & 1* \\
\hline $\begin{array}{l}\text { No } \\
\text { pollen }\end{array}$ & 22 & 4 & 8 & $13^{\#}$ & $18^{*}$ & 3 & 13 & 5 & 1 & 5 \\
\hline
\end{tabular}

a Establishment is roughly defined as the 196 most abundant OTUs in the combined hindgut tissues.

934 *Treatment significantly different from the colony control based on a test for proportions $(\mathrm{p}<0.00001)$.

935 \# Treatment significantly different from fresh pollen and colony control based on a test for proportions ( $\mathrm{p}<0.01)$. 


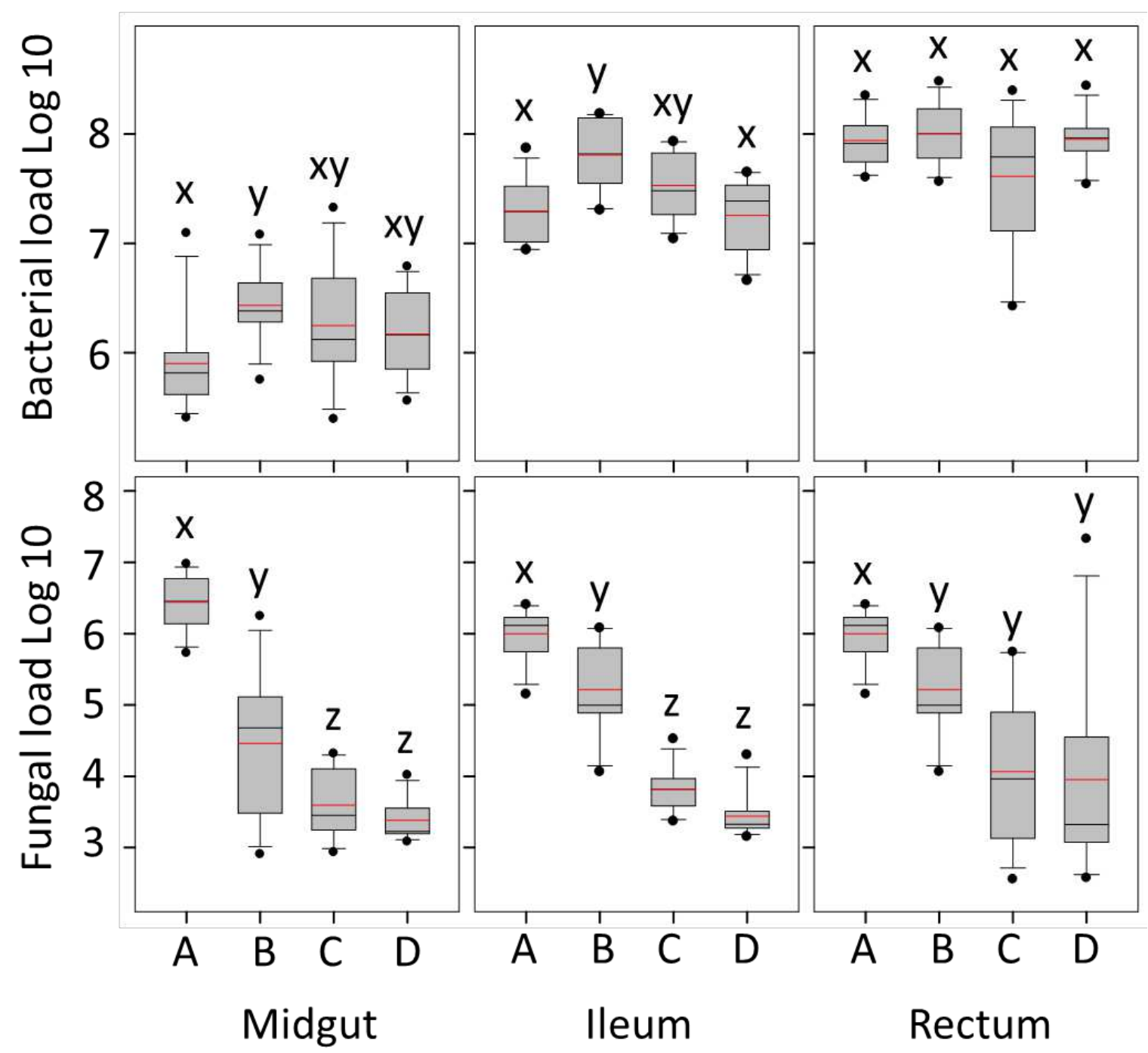

945

946

947

948

949

950

951

952

953

954

955

956

957

958

959
Figure 1. Bacterial and fungal load differ by social context and diet throughout the gut. On the $x$-axis, treatments, $(A, B, C, D)$, are each composed of three replicate cages or colonies. $A$ is the positive control; a natural and vibrant colony environment. Treatments $B, C$, and $D$ lack the colony context, and consist of cages containing 300 newly emerged bees and wax combs containing each diet treatment; B: Fresh stored pollen (beebread), C: Autoclaved corbicular pollen, and D: No pollen (negative control). Values were determined by bactquant and fungiquant and are cell number for bacteria, rRNA copy number for fungi. 


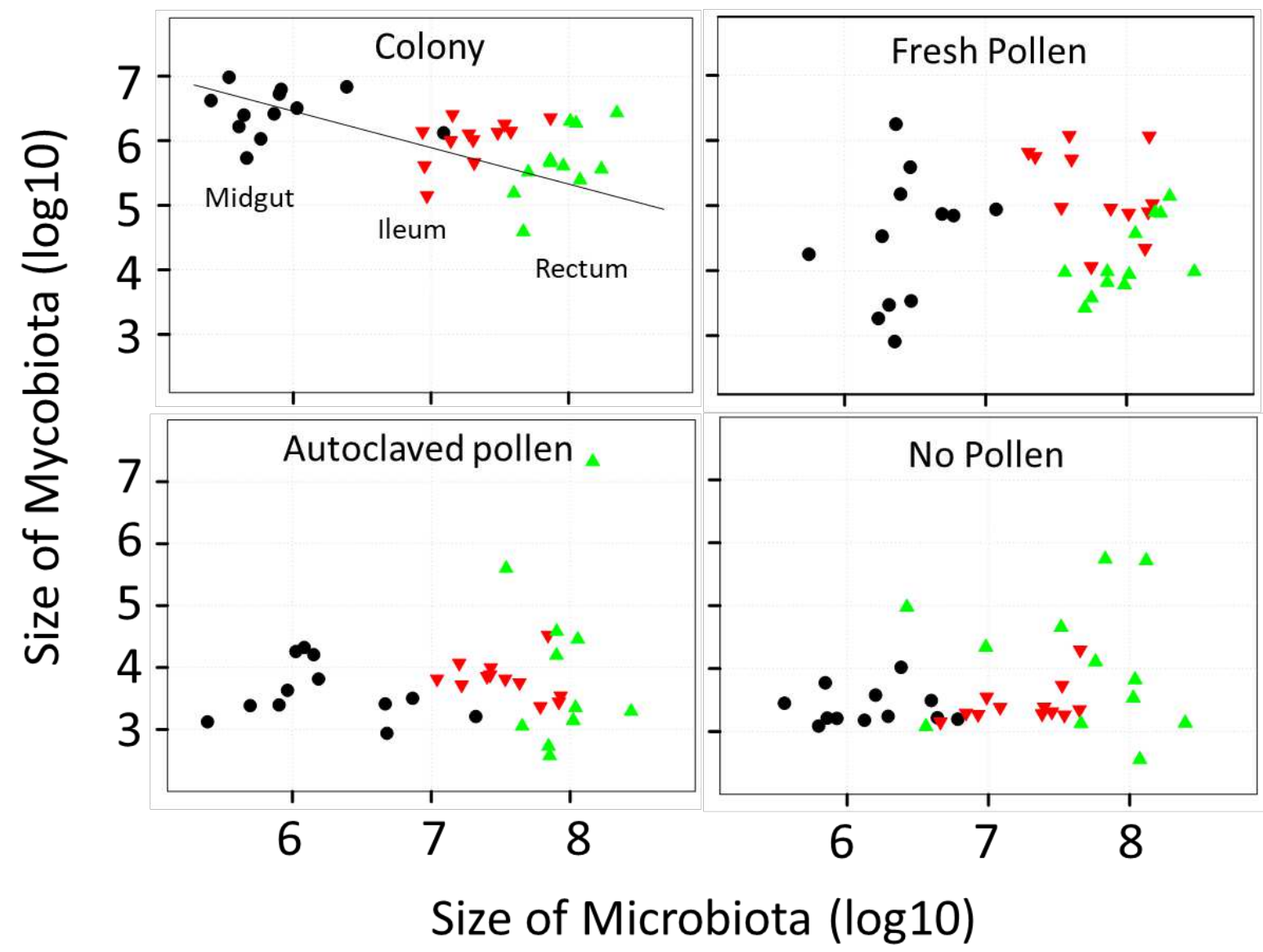

961

962

963

964

965

966

967

968

969
Figure 2. Throughout the alimentary tract, the presence of fungi (the mycobiome) or fungal associated factors was correlated with the structure of the microbiome. In our positive control, colony reared bees, there is a positive association of microbiota and mycobiota size, but considering the alimentary tract as a unit, fungal load decreases concurrent with increasing bacterial load ( $\mathrm{Rsq}=0.25, \mathrm{~F}=11.4503, \mathrm{p}=0.002$ ). This pattern shifted significantly when workers were detached from the active hive environment and provided various diet treatments. Values are from Figure 1. 

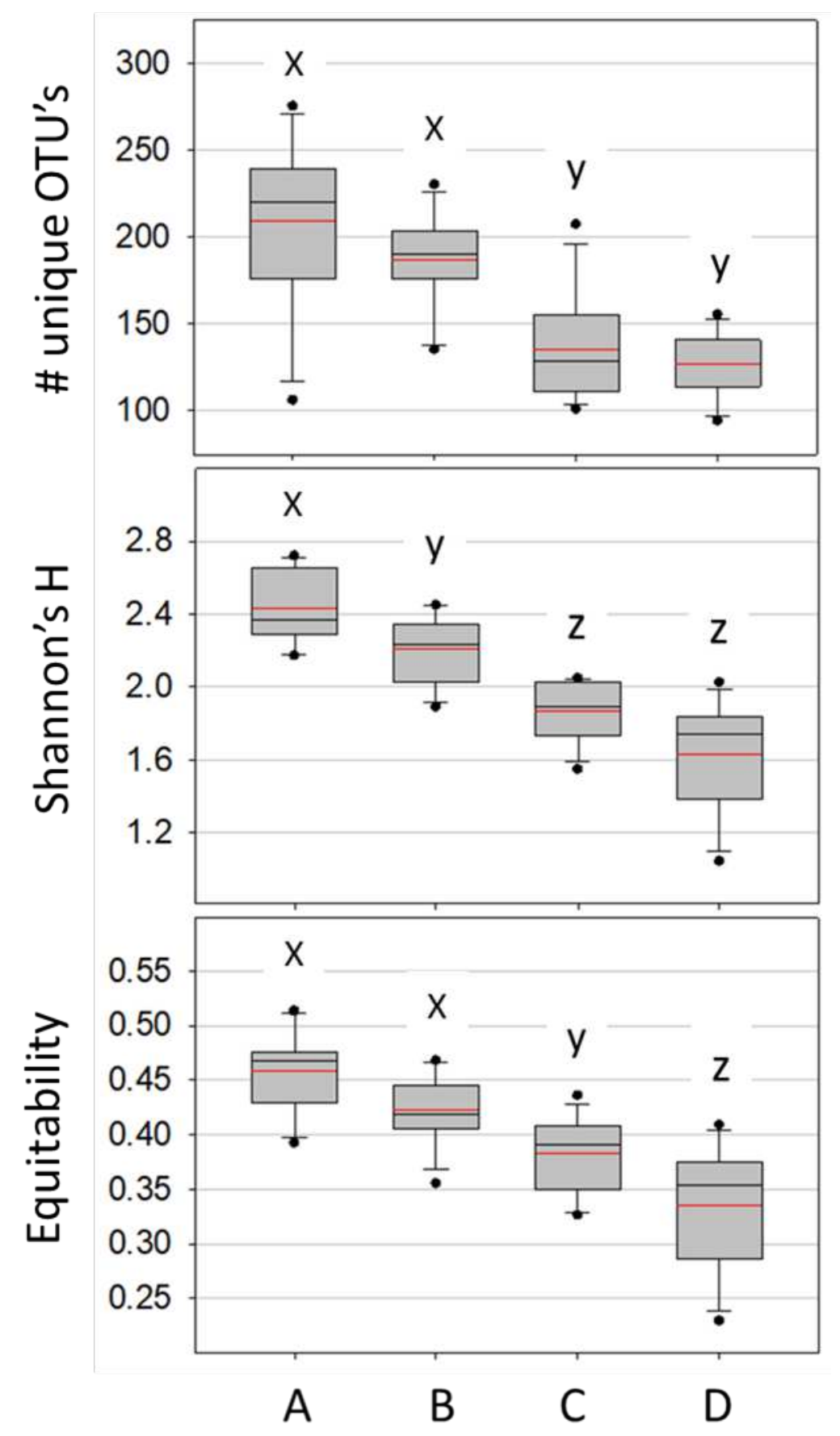

972 Figure 3. Diversity of combined hindgut tissues (ileum and rectum) based on unique bacterial 973 OTUs (top panel), Shannon's $\mathrm{H}$, and Equitability, a measure of evenness. The treatments on the $974 x$-axis are colony control (A), fresh pollen (B), autoclaved pollen (C), and no pollen (D). Within 975 each panel, distributions (boxplots) with the same lower case letter do not differ significantly ( $p$ $976<0.05)$ based on a t-test. 


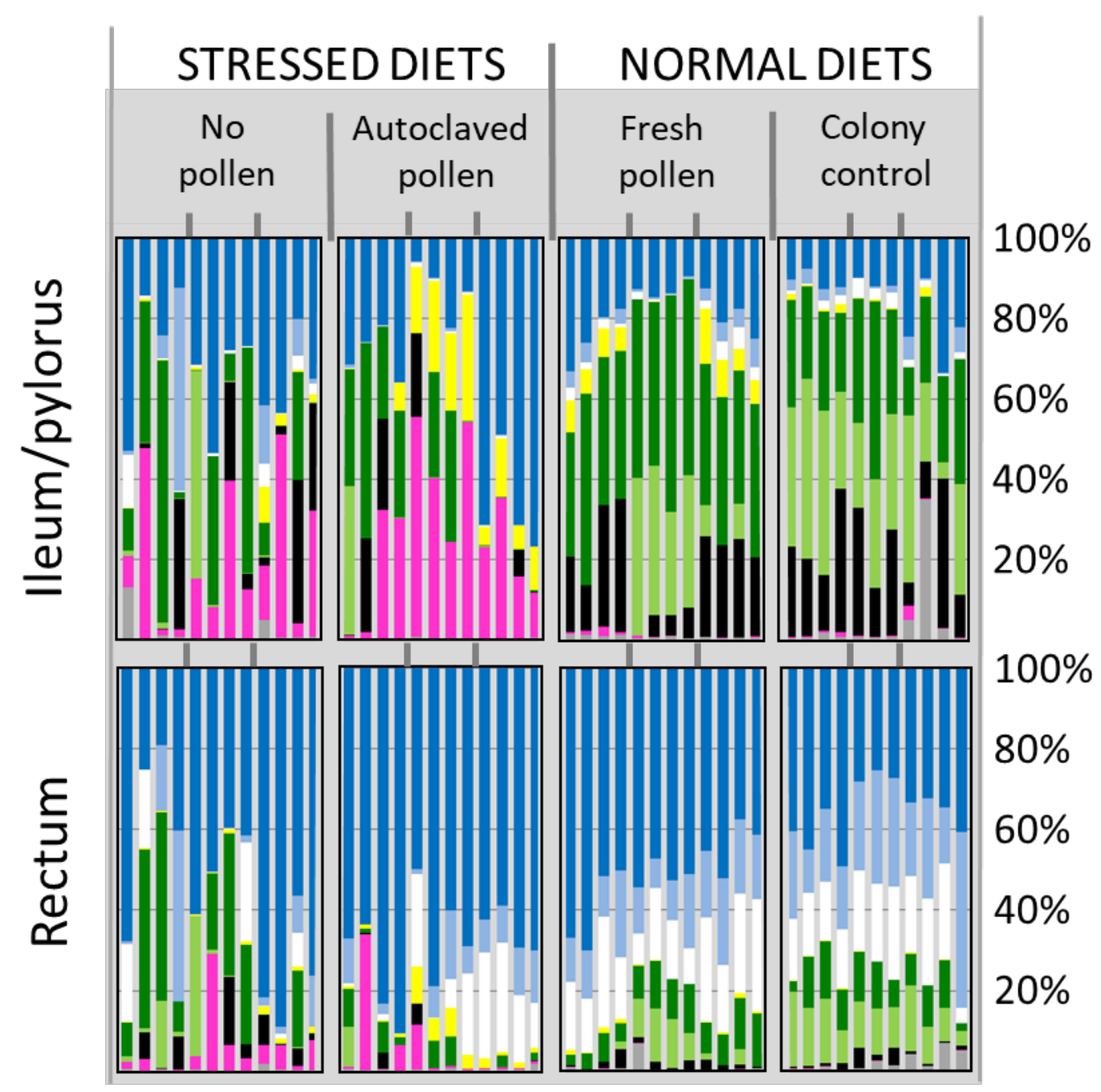

980

981

982

983

984

985

986

987

988

989

990

991

992

993

994

995

996 $\underline{\text { Taxonomic color key }}$

Figure 4. Bar charts representing relative abundance of bacterial OTUs from two hindgut tissues and four treatment conditions, each composed of three replicate cages or colonies shown at the top of the diagram. The colony control is a natural and vibrant colony environment. Diet treatments lack the colony context, and consist of cages containing 300 newly emerged bees and wax combs containing each diet treatment. All colonies were provided autoclaved sucrose syrup and water ad libitum. The vertically oriented ileum and rectum microbiotas correspond to the same worker individuals. Note replicate variation.
Lactobacillus Firm5 Lactobacillus Firm4 Bifidobacterium asteroides Lactobacillus kunkeei Snodgrassella alvi Gilliamella apicola Frischella perrara Bombella apis Others 
Ileum/pylorus

Rectum

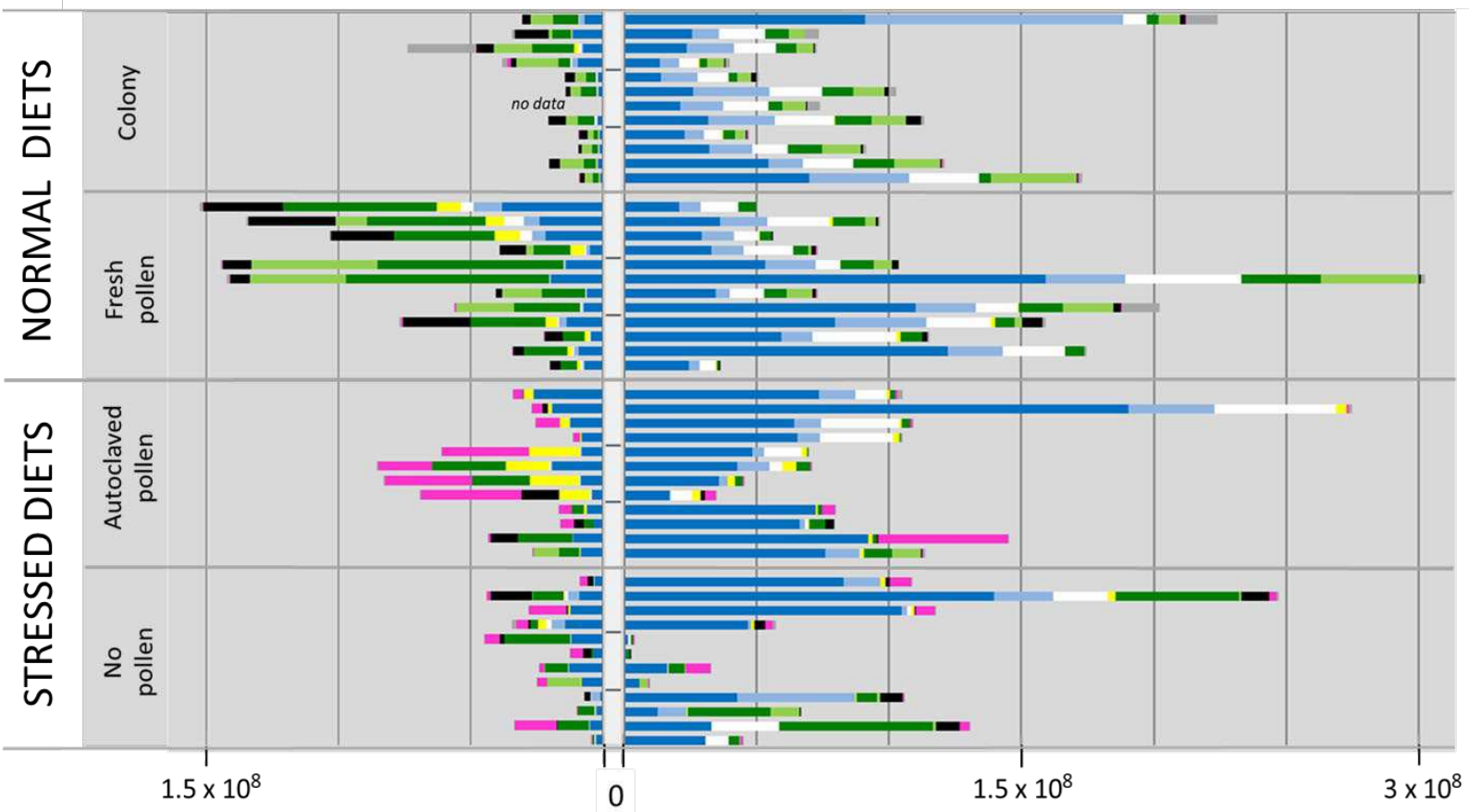

Figure 5. Microbiota of the hindgut of 9-Day old workers reared in large functional colonies or in social isolation with different diet treatments. Bar charts represent the absolute abundance of bacterial OTUs from two hindgut tissues (ileum/pylorus and rectum). Colony is the positive control; a natural and vibrant colony environment. The three diet treatments lack the colony context, and are associated with significantly different diet consumption. Microbiota size in the ileum was significantly associated with hindgut weight across the diet treatment groups. Each replicate treatment was comprised of 300 newly emerged bees and wax combs containing ad libitum diet

$\underline{\text { Taxonomic color key }}$

1013

1014 treatment. The $x$-axis is centered at zero and

1015

1016

1017

1018

1019

1020 increases in absolute value moving in either direction.
Lactobacillus Firm5

Lactobacillus Firm4 Bifidobacterium asteroides Lactobacillus kunkeei

Snodgrassella alvi Gilliamella apicola Frischella perrara Bombella apis Others 

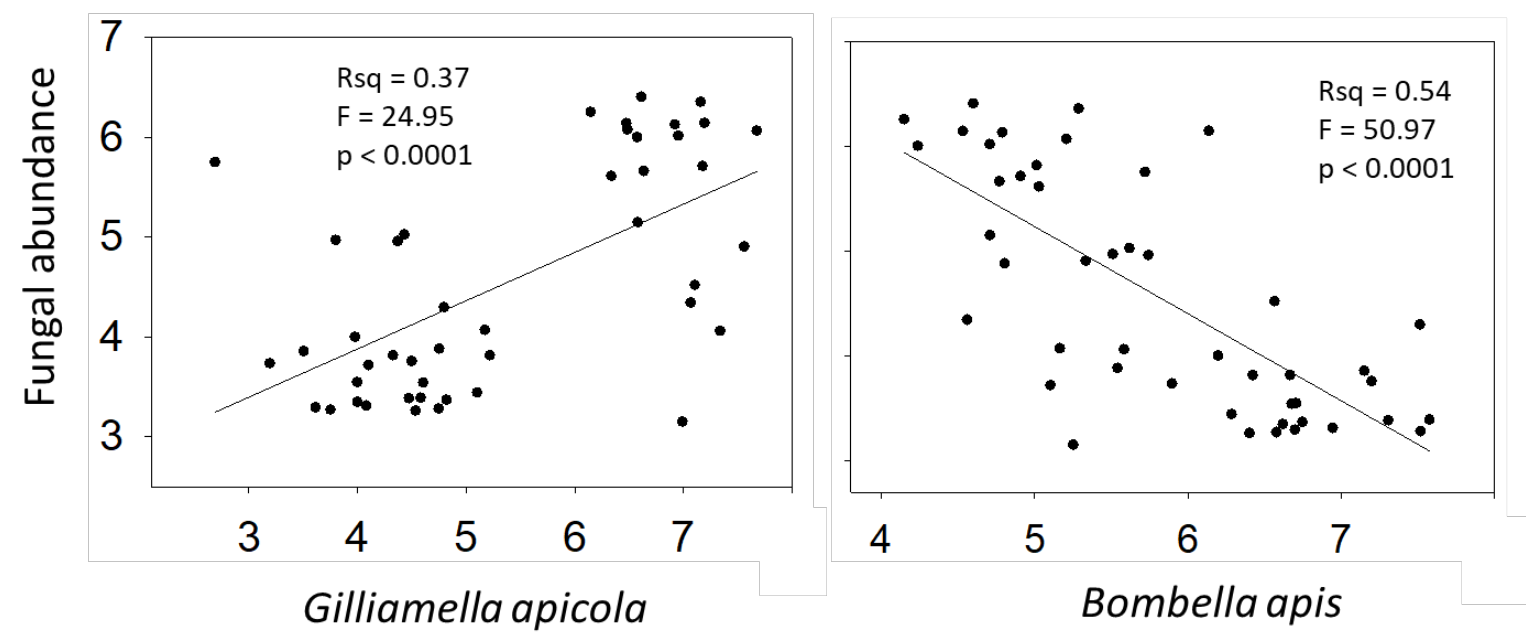

1022

1023 Figure 6. The significant relationship of fungal abundance with two different bacteria in the 1024 worker ileum. Trend line represents Pearson's correlations of log transformed abundance 1025 measures. Axes are displayed as (log 10) exponents. Fungi are $18 \mathrm{~S}$ rRNA copy number, and 1026 bacteria represent cell counts, normalized by genome specific copy number. 


\section{Supplementary Files}

This is a list of supplementary files associated with this preprint. Click to download.

- TableS1.990TUTaxonomy.xlsx

- TableS2.AbsoluteabundanceANOVAandMANOVAstatistics.xlsx 